DOI: 10.30842/ielcp230690152417

Natalija Ganina

(Moskauer Staatliche Lomonossov-Universität)

\title{
DIE VERWANDTSCHAFTSTERMINOLOGIE IM LÜBISCHEN RECHT
}

\author{
The kinship terms in the Lübeck Law
}

This study focuses on the kinship terms in the Lübeck City Law from the lexical point of view. The following points are assessed: the structure of this lexical group (kinship by blood, kinship by marriage, and kinship in general); etymological parallels and Germanic isoglosses of the terms; their semantics and specifics of the use in the Lübeck Law. The study is based on the text presented in the principal manuscript of the Lübeck Law, namely, in the 'Codex of the Lübeck Chancellery' (Kiel, Stadtarchiv, Hs. 79413, c. 1280), considering other manuscripts of the Lübeck Law.

Key words: Lübeck, Lübeck Law, Middle Low German, kinship terms, Lübeck Law manuscripts.

Н. А Ганина

(МГУ им. М. В. Ломоносова)

\section{Термины родства в любекском праве}

В статье, посвященной исследованию лексики любекского городского права, рассматривается функционирование субстантивных обозначений времени и производных от них наречий. В частности, определяется состав этой лексико-семантической группы, обсуждается семантика и правовая специфика соответствующих обозначений. Исследование основывается на тексте, представленном в важнейшей рукописи любекского права, а именно в «кодексе любекской канцелярии» (Киль, Stadtarchiv, Hs. 79413, около 1280 г.), тогда как при обсуждении вступлений, колофонов и дополнений учитываются и другие кодексы любекского права, прежде всего недавно обнаруженный кодекс Бардевика (Юрьевец, «Музеи города Юрьевца», ЮКМ-2010, 1294 г.).

Ключевые слова: Любек, любекское право, средненижненемецкий, Кильский кодекс, кодекс Бардевика.

Die Verwandtschaftsbezeichnungen gehören zum traditionellen Wortschatz der Rechtstexte, was vor allem durch das Erbrecht bedingt wird. Im altgermanischen Recht treten die Verwandtschaftsbezeichnungen auch im Zusammenhang mit gesetzlicher Bestimmung des Wergelds hervor, weil die Entschädigung an die nächsten männlichen Verwandten des Geschädigten ging. Das Wergeld ist für das lübische Recht nicht mehr aktuell, während das Erbrecht sehr ausführlich ausgearbeitet ist und, wie bei Wilhelm Ebel hervorgehoben wird, im Gang der Rechtsgeschichte als die „Mutter des 
Eigentums“ erscheint (Ebel 1980: 7). Die Hauptaufgabe der Erforschung der Verwandtschaftsterminologie im lübischen Recht aus lexikalischer Sicht ist die Klärung der Frage, in welchem Maße es das altgermanische System repräsentiert. Unter dem System der Verwandtschaftsbezeichnungen im lübischen Recht wird das entsprechende mittelniederdeutsche System verstanden, das in der Schreibsprache (Nordniederdeutsch) bzw. im Statutenbestand des lübischen Rechts zum Ausdruck kommt. Dabei wird uns sowohl der Bestand der lexikalischen Gruppierung bzw. germanische Isoglossen der Einzelglieder (ohne auf die gut untersuchte indogermanische Etymologie der Hauptglieder zu kommen) als auch der Wortgebrauch im lübischen Recht interessieren.

Eine anschauliche Rekonstruktion des urgermanischen Systems der Verwandtschaftsbezeichnungen wird im Beitrag von Winfred P. Lehmann zum indogermanischen sozialen und wirtschaftlichen Erbwortschatz bzw. zu seinem Ersatz im Urgermanischen geboten (Lehmann 1968: 14-17). Besonders wichtig ist die Schlussfolgerung, dass die Veränderungen im altgermanischen System in der stärkeren Gliederung der Verwandtschaftsbezeichnungen bestehen, wobei auch germanische Neubildungen als zusätzliche Terminologie hinzukommen (Lehmann 1968: 15, 25). Das von Lehmann gebotene Modell wird im Folgenden bei Erörterung der Verwandtschaftsbezeichnungen im lübischen Rechts berücksichtigt.

Das System der Verwandtschaftsbezeichnungen im lübischen Recht lässt sich folgendermaßen darstellen.

\section{Blutsverwandtschaft}

vader (m.) 'Vater' (Ki 19, 23, 33, 128, Ki, Register, Artikeltitel 19, 113) - vgl. got. fadar (1x in der gotischen Bibel), an. faðir, ae. fader, afries. fader, as. fadar, ahd. fatar 'Vater' (Lehmann 1986: 101) gegen got. atta 'Vater', siehe dazu (Lehmann 1986: 46);

môder (m.) 'Mutter' (Ki 19, Ki, Register, Artikeltitel 113) vgl. an. móðir, ae. mōdor, afries. mōder, as. môdar, ahd. muotar (de Vries 1962: 391; Kluge 1999: 577) gegen got. aipei 'Mutter', siehe dazu (Lehmann 1986: 20);

oldere (öldere) (Pl.) 'Eltern' (Ki 94, 167, 250, Ki, Register, Artikeltitel 12, 15, 20, 166, 193) - vgl. ae. eldran, yldran, as. eldiron, aldiro, ahd. eltiron (altiron) aus westgerm. *aldizōn, Pl. des Komparativs von 'alt', auch in ae. eldran, yldra, afries. alder, elder (Kluge 1999: 218) und an. forellrar, foreldri, forellri 'Eltern' (de Vries 1962: 138) gegen got. berusjos 'Eltern', siehe dazu (Lehmann 1986: 66f.); 
kint (n.) 'Kind', Pl. kindere (Ki 4-7, 12-15, 21, 23f., 33, 77, 94, 108, 114, 131, 157, 173, 187, 195, 200, 221, 240) — vgl. ahd. kind gegen ae. cild 'Kind' und germ. *barnan, got. barn, krimgot. baar, an. barn, ae. bearn, afries. bern, as., ahd. barn 'Kind'; zum Letzteren siehe (Lehmann 1986: 62). Da as. kind und afries. kind aus dem Hochdeutschen entlehnt sind (Kluge 1999: 442), handelt es sich auch bei mnd. kint um hochdeutsche Entlehnung.

sone (söne) (m.) 'Sohn' (Ki 1, 128, 154, 221, 227, Ki, Register, Artikeltitel 231) - vgl. got. sunus, an. sonr, ae., afries., as., ahd. sunu 'Sohn'.

dochter (f.) 'Tochter' (Ki 1, 37, 221, 227, 235, Ki, Register, Artikeltitel 231) - vgl. got. dauhtar, an. dóttir, ae. dohtor, afries. dochter, as. dohtar, ahd. tochter 'Tochter'.

brôder (brûder) (m.) 'Bruder' (Ki 128, 167, 227, 235, Ki, Register, Artikeltitel 217, 223) - vgl. got. bropar, krimgot. bruder, an. bróðir, ae. brōpor, afries. brother, as. brôðar, ahd. bruoder 'Bruder'.

suster (süster) (f.) 'Schwester' (Ki 167, 227, 235, Artikeltitel 217) - vgl. got. swistar, krimgot. schuuester, an. systir, ae. sweostor, afries. swester, suster, as., ahd. swestar.

halfbrōder (halfbrûder) (m.) 'Halbbruder' (Ki 19, 221), halfsuster (f.) 'Halbschwester' (Ki 19, 221) — vgl. an. hálfbróðir 'Halbbruder', hálfsystir 'Halbschwester' (Cleasby, Vigfusson 1957: 82, 615). Dagegen vgl. mhd. halpbruoder 'Laienbruder' ${ }^{1}$ und halpschwester 'halbe Ordensschwester' (im Deutschen Orden) neben mhd. stiefbruoder 'Halbbruder', stiefswester 'Halbschwester' (Benecke, Müller, Zarncke 1854-1866, 2/2: Sp. 776a; Lexer 18721878, 2: Sp. 1153). Zu germ. *steup-/stiup- 'beraubt' in Zusammensetzungen mit Verwandtschaftsnamen (an. stjúp-, ae. stēop-, afries. stiāp-, ahd. stiof-, mhd. stief-, mnd. stêf-) siehe (Kluge 1999: 795).

eldervader (ölder-) (m.) 'Großvater' (Ki 157, Ki, Register, Artikeltitel 156), eldermoder (ölder-) 'Großmutter' (Ki 157, Ki, Register, Artikeltitel 156) - vgl. ae. ealdefader 'Großvater', ealdfader 'Großvater, Ahn', ealde-ealdefader 'Urgroßvater', eald(e)mōder 'Großmutter', afries. ald(e)fader 'Großvater', aldmōder 'Großmutter' (Bosworth, Toller 1898: 227f.; Toller 1921: 166) und mhd. eltervater 'Großvater' (seit dem 12. Jh.), eltermuoter

${ }^{1}$ Das mhd. halpbruoder fehlt bei Lexer; siehe Angaben im Belegarchiv des 'Mhd. Wörterbuch online': http://www.mhdwb-online.de/wb.php? buchstabe $=$ H\&portion=160\&link_lid=246628700\#246628700 (letzter Aufruf 05.03.2020) mit Verweis auf Nikolaus von Jeroschin 14840 (Nikolaus von Jeroschin 1861). 
(seit dem 13. Jh.) 'Großmutter' (Kluge 1999: 218) gegen an. afi 'Großvater', ái 'Urgroßvater', got. awo 'Großmutter', vgl. lat. avus 'Großvater, Ahn', av(i)a 'Großmutter', siehe dazu (Lehmann 1986: 53), auch unten $\mathrm{zu}$ mnd. om. Weiterhin $\mathrm{zu}$ den altnordischen Bezeichnungen für 'Großvater' und 'Großmutter' bzw. 'Urgroßvater', und 'Urgroßmutter' vgl. an. föðurfaðir 'Großvater väterlicherseits', móðurfaðir 'Großvater mütterlicherseits', föðurmóðir 'Großmutter väterlicherseits', móðurmóðir 'Großmutter mütterlicherseits', föðurafi 'Urgroßvater väterlicherseits', móðurafi 'Urgroßvater mütterlicherseits' (Cleasby, Vigfusson 1957: 137, 435), an. amma 'Großmutter' (in den Sagas selten belegt), langamma 'Urgroßmutter', móðuramma 'Urgroßmutter mütterlicherseits' (Cleasby, Vigfusson 1957: 19, 435) zu ahd. ammâ

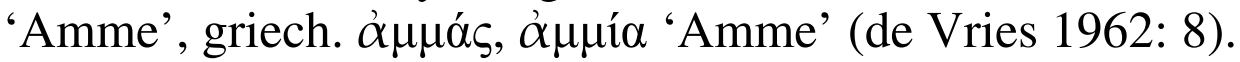

soneskint (söneskint) (n.) 'Sohneskind', dochterkint (n.) 'Tochterkind' (Ki 235, Ki, Register, Artikeltitel 231) — zu germanischen Zusammensetzungen für 'Enkel' vgl. an. sonarsonr 'Sohnessohn', sonardóttir 'Sohnestochter', dóttursonr 'Tochtersohn', dótturdóttir 'Tochtertochter' und barnabörn (Pl.) 'Enkelkinder' (Cleasby, Vigfusson 1957: 52, 102, 578) neben afries. bernbern 'Kindeskind' (von Richthofen 1840: 627), ae. sunsunu 'Sohnessohn' und dohtorsunu 'Tochtersohn' (Toller 1921: 154; Bosworth, Toller 1898: 937) gegen spätahd. eniklîn, enichlin, mhd. eninkel 'Enkel', Diminutiv zu ahd. ano 'Ahn', siehe dazu (Kluge 1999: 221f.). $\mathrm{Zu}$ mnd. dochterkint vgl. auch mhd. tihter, tîhter, diechter als Diminutivbildung auf $\bar{l}$ in der Bedeutung 'Tochterkind' mit solchen indogermanischen Parallelen wie aind. dauhitra- und gr.

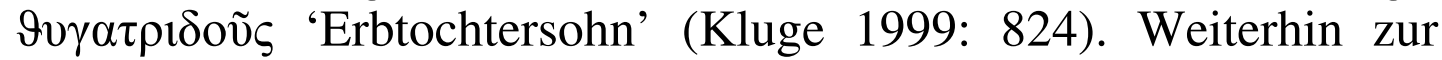
Entwicklung der germanischen Terminologie für 'Enkel' siehe (Haubrichs 2001).

om (m.) 'Mutterbruder' (Ki 157, 174) — vgl. ae. ēām, afries. èm, ahd. ôheim (seit dem 9. Jh.), mhd. aheim(e), mndl. oom 'Mutterbruder', nhd. Oheim 'Onkel' (veraltet bzw. mundartlich $\mathrm{Ohm}$ (m.), ursprünglich niederdeutsch), aus westgerm. *awa-haimaz 'Mutterbruder' (Kluge 1999: 598). Weiterhin vgl. got. awo 'Großmutter', lat. avunculus 'Onkel, Mutterbruder', lit. avýnas 'Mutterbruder', aksl. yŭ, aruss. (в)yŭ 'Mutterbruder' (Lehmann 1986: 53) bzw. got. haims (f.) 'Dorf', anahaim(ei)s 'heimisch', 'daheim', an. heimr 'heim', 'Welt', ae. hàm, ne. home, afries. hām, hèm, as. hêm, ahd. heim 'heim, Zuhause', vgl. lit. šeimà, lett. saime 'Familie', aruss. стьмиц, russ. семья 'Familie' (Lehmann 1986: 170). Dagegen vgl. an. móðurbróðir 'Mutterbruder' neben afabróðir 
'Großonkel väterlicherseits' und ömmubróðir 'Großonkel mütterlicherseits' (Cleasby, Vigfusson 1957: 82).

Im lübischen Recht kommt das Wort om in der ursprünglichen archaischen Bedeutung 'Mutterbruder' vor, vgl. die Paarformeln de om vnde veddere vnde vadhe. vnde moddere (Ki 157) 'der Mutterbruder und der Vaterbruder sowie die Vaterschwester und die Mutterschwester', om oder veddere iof vade oder meddere (Ki 174) 'der Mutterbruder oder der Vaterbruder bzw. die Vaterschwester oder die Mutterschwester'.

veddere (m.) 'Vaterbruder' (Ki 157, 174) - vgl. ae. fadera, afries. federia, fidiria, fedria, ahd. fetiro, fatureo 'Vaterbruder' aus

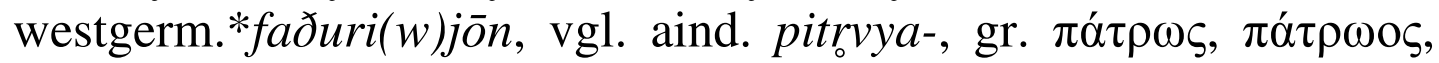
lat. patruus und weitere indogermanische Parallelen (Kluge 1999: 863; Lehmann 1986: 101; von Richthofen 1840: 730). Dagegen vgl. an. föðurbróðir 'Vaterbruder' als germanische Neubildung (Cleasby, Vigfusson 1957: 82). Obwohl das Wort veddere im Mittelniederdeutschen in den Bedeutungen 'Vaterbruder', 'Brudersohn' gebraucht wird, vgl. (Schiller, Lübben 1875-1881, 5: 217; Lasch, Borchling 1956-2007, 1: Sp. 674), kommt veddere im lübischen Recht nur in der Bedeutung 'Vaterbruder' vor. ${ }^{2}$ Dies kann sowohl durch die oben anfgeführten Paarformeln (Ki 157, 174) mit den Parallelbezeichnungen der Onkel und Tanten mütterlicherseits und väterlicherseits als auch durch die Textstelle de om vnde veddere. vnde vadhe. vide moddere ofte ere kindere. 'der Mutterbruder und der Vaterbruder sowie die Vaterschwester und die Mutterschwester und ihre Kinder' (Ki 157) bestätigt werden, auf die sich die Gebrauchsfälle von veddere im lübischen Recht beschränken.

vade (f.) 'Vaterschwester' (Ki 157, 174) - vgl. ae. faðu, faðe 'Vaterschwester', 'Tante' (Bosworth/Toller 1898: 271; Toller 1921: 206) gegen as. wasa, ahd. basa, mhd. base 'Vaterschwester', seit dem 15. Jh. 'Tante', danach auch 'Nichte' (selten), nhd. Base 'Kusine', 'entfernte weibliche Verwandte' (Kluge 1999: 83) und an. föðursystir 'Vaterschwester' (Cleasby, Vigfusson 1957: 615). Dass vade im lübischen Recht 'Vaterschwester' bedeutet, wird durch die Paarformel vadhe. vnde moddere 'Vaterschwester und Mutterschwester' (Ki 157), vade oder meddere 'Vaterschwester oder Mutterschwester' (Ki 174) bezeugt, die Entsprechung in ae. faðu oððe mōddrie hat (Homl. Th. ii. 94, 32; Bosworth/Toller 1898: 271).

\footnotetext{
2 'Brudersohn, Vaterbruder' bei (Korlén 1951: 202) wohl mit Stütze auf die Hauptbedeutung 'Vetter' bei (Schiller, Lübben 1875-1881, 5: 217).
} 
moddere (meddere) (f.) 'Mutterschwester' (Ki 157, 174) — vgl. ae. mōddrie ( $m \bar{o} d(d)$ rige, mōderge), 'Mutterschwester', auch 'Kusine' (Bosworth/Toller 1898: 695) gegen ahd. muоma, mhd. muome 'Mutterschwester', nhd. Muhme 'Tante', 'Kusine', 'weibliche Verwandte' (Kluge 1999: 573) und an. móðursystir 'Mutterschwester' (Cleasby, Vigfusson 1957: 615). Weiterhin zu ahd. muoma vgl. an. móna 'Mutter', 'Amme' (de Vries 1962: 392; Cleasby, Vigfusson 1957: 435).

nichte 'Nichte' (Ki 137) - vgl. an. nipt 'weibliche Verwandte', 'Nichte', ae. nift 'Nichte', 'Enkelin', 'Stieftochter', afries. nift 'Nichte', ahd. nift(a), niftila, neftil, mhd. niftel(e) 'Nichte', nhd. Nichte (seit dem 16. Jh., aus dem Niederdeutschen) aus germ. *nefti'weibliche Verwandte', 'Nichte', vgl. aind. naptī 'Tochter', 'Enkelin' neben idg. *nepōt 'Enkel', später auch 'Neffe', vgl. lat. nepōs, Gen. nepōtis 'Enkel', 'Neffe' und weitere indogermanische Parallelen (Cleasby, Vigfusson 1957: 455; de Vries 1962: 410; Bosworth, Toller 1898: 719; von Richthofen 1840: 951; Kluge 1999: 587). Das entsprechende Maskulinum aus germ. *nefōn 'Neffe' kommt im lübischen Recht nicht vor, weil es keine Rechtskollisionen derart vorhanden sind.

susterkint (süsterkint) (n.) 'Schwesterkind' (Ki 174) — zum Modell vgl. an. systurbarn (Cleasby, Vigfusson 1957: 615).

\section{Verwandtschaft durch Heirat}

man (m.) 'Mann' (Ki 1, 2 u. ö.), 'Mann, Ehemann' (ab Ki 5) vgl. got. manna, ai. maðr, mannr (selten), ae. mon(n), (man(n)), afries. mon, man, as. mann, ahd. $\operatorname{man}(n)$ 'Mann', 'Mensch' (Lehmann 1986: 244; Cleasby/Vigfusson 1957: 407f.; de Vries 1962: 374f.; Bosworth/Toller 1898: 668; von Richthofen 1840: 932f.); in der Bedeutung 'Ehemann' vgl. afries. mon, ahd. man (n) gegen got. $a b a$ (Lehmann 1986: 1), an. verr (Cleasby, Vigfusson 1957: 698f.; de Vries 1962: 657), ae. wer 'Mann', 'verheirateter Mann' (sozialer Status), 'Ehemann', ceorl 'Mann', 'Ehemann', ceorlian (schw. V. 2) 'heiraten (für Frauen)' im Gegensatz zu wïfian (schw. V. 2) 'heiraten (für Männer)', (Bosworth, Toller 1898: 151, 1205) und weiterhin an. húsbóndi, ae. hüsbonda (aus dem Skandinavischen) 'Hausherr', 'Mann' (nur selten) (Cleasby, Vigfusson 1957: 294; Bosworth, Toller 1898: 568), me. husbonde (hosbonde) 'Ehemann', ne. husband 'Ehemann'.

wîf (n.) 'Frau' (Ki 2, 5, 7, 11-15, 21, 42, 77, 101, 108, 114, 122, 148, 163, 172, 178, 187, 195), 'Ehefrau' (ab Ki 5) - vgl. an. víf 'Frau' (poetisch, selten), ae., afries. wïf, ahd. wîp 'Frau', 'Ehefrau' 
als germanische Neubildung (Cleasby, Vigfusson 1957: 714; Kluge 1999: 879f.) gegen got. qino 'Frau', an. kona 'Frau', 'Ehefrau', ae. cwēne 'Frau', 'Ehefrau', 'Dienerin', 'Dirne', ne. quean (arch.) 'Prostituierte', as., ahd. quena 'Ehefrau' und got. qens 'Frau, Gattin', an. kván, kvoén 'Frau, Gattin' . ae. cwēn 'Frau', ne. queen, as. quân 'Ehefrau', vgl. aind. jáni-, aksl., aruss. жена 'Frau', 'Ehefrau' und weitere indogermanische Parallelen (Lehmann 1986: 274, 277). Die Bedeutung 'weibliche Person' wird im lübischen Recht auch durch mnd. wîvesname (m.) wiedergegeben: So we begrepen. oder gevangen wert mit iemendes dochter. oder nichten. oder mit ienegeme wiuesnamen ( $1 \mathrm{x}, \mathrm{Ki} 137)$. Vgl. mhd. wîbes name, nhd. Weibsen (regional; seit dem 17. Jh.) (Kluge 1999: 880).

hûsfrûwe (f.) 'Hausfrau', 'Ehefrau' (Ki 200, 201) - vgl. an. húsfreyja, anorw. hüsproya, hūsprei, aschw. husprea, agotl. húsfroyja 'Hausfrau', 'Ehefrau', mhd. hûsvrouwe (hûsvrowe, hûsvrou), wobei nschw., ndä. hustru aus dem Mittelniederdeutschen entlehnt ist (de Vries 1962: 268), zu mhd. hûsvrouwe siehe (Lexer 1872-1878, 1: Sp. 1407).

swager (m.) 'angeheirateter Verwandter' (Ki 135f., 208) — vgl. mhd. swâgur, swâger 'Schwager', weiterhin got. swaihro, an. svara, ae. sweger, ahd. swigar 'Schwiegermutter', got. swaihra, aschw. svér, svaer (dagegen an. verfaðir 'Schwiegervater'), ae. swehor, swēor, afries. swiār, ahd. swehur 'Schwiegervater', mhd. sweher, nnd. Schwäher 'Schwiegervater', 'Schwager' aus germ. *swegurōn- (mit der Sonorisierung des Reibelautes nach dem Vernerschen Gesetz) 'Schwiegermutter', *swehuran- 'Schwiegervater', *swēgura- (mit Dehnstufe) 'zur Sippe des Schwiegervaters gehörend', vgl. aind. śváśura-, aksl., aruss. свекры 'Schwiegermutter, Mutter des Mannes', lat. socer 'Schwiegervater' und andere indogermanische Parallelen (de Vries 1962: 571; Lehmann 1986: 332; Kluge 1999: 747, 751).

Im lübischen Recht wird mnd. swager in der Bedeutung 'angeheirateter Verwandter' in der Paarformel maghe \& swagere 'Blutsverwandte und angeheiratete Verwandte' (2x, Ki 135f., swaghere vnde maghe $1 \mathrm{x}$, Ki 208) gebraucht, vgl. So wanne men $n$ men schal iemene van der louen to deme rade. de gene de ene nomet. de schal gan van deme hus vnde alle de gene de sine maghe. vnde sine swagere sint. So kommt mnd. swager im lübischen Recht nur im Plural vor, wobei das Singular nicht belegt ist und die Bedeutung 'Schwager' nicht explizit zum Ausdruck kommt.

wedewe (f.) 'Witwe' (Ki 10, 131); wedewere (weduwere) (m.) 'Witwer' (Ki 131) — vgl. got. widuwo, ae. widewe, afries. widwe 
(wedwe, wide), as. widowa, ahd. wituwa, vgl. aind. vidhávā, lat. vidua, aksl., aruss. вьдова und weitere indogermanische Parallelen (Lehmann 1986: 402) gegen an. ekkja 'Witwe' aus germ. *ainakjōn (de Vries 1962: 99). Es wird hervorgehoben, dass das Femininum als Grundlage der Bezeichnungen weiterer Verhältnisse genommen worden ist, wie z. B. got. widuwairna 'Waise' und nhd. Witwer (Kluge 1999: 895). Zu got. widuwairna siehe (Lehmann 1986: 402). Das Maskulinum 'Witwer' wird in den altgermanischen Sprachen nach üblichen Wortbildungsmodellen von Nomina agentis gebildet, vgl. ae. widuwa (m. -n) 'Witwer', mhd. witewer, witwer (Bosworth, Toller 1898: 1217; Lexer 1872-1878, 3: Sp. 953).

Die Bezeichnungen von 'Schwiegervater, -mutter' bzw. 'Schwiegersohn, -tochter' fehlen im lübschen Recht, weil es keine darauf bezogene Rechtskollisionen gibt. Ebenfalls fehlen die Bezeichnungen von 'Braut' bzw. 'Bräutigam', wobei nur im Oldenburger Codex (Schleswig, Landesarchiv, Bestand 400.3 Oldenburg Nr. 14, früher Oldenburg, Stadtarchiv, ohne Signatur) die Zusammensetzung brûtschat 'Mitgift' (O 18) belegt ist.

\section{Verwandtschaft im allgemeinen Sinne}

mach (m.) 'Blutsverwandter' (Ki 27, 114, 135f., 208, 229) -

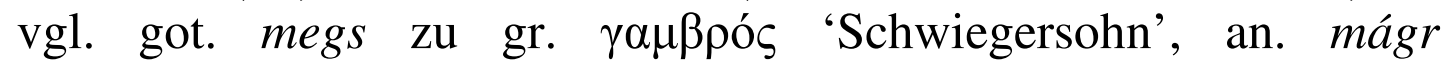
'Verwandter durch Heirat; Schwager, Schwiegersohn, Schwiegervater', ae. $m \bar{x} g$ (m.) 'Verwandter', $m \bar{x} g$ (f.) 'Verwandte', afries. $m \bar{e} c h$, mei, as. mâg, ahd. mâg 'Verwandter' als germanische Neubildung ohne sichere indogermanische Etymologie, wahrscheinlich aus idg. *megh- 'lieb haben, mögen', 'freundlich sein' mit Dehnstufe (Cleasby, Vigfusson 1957: 415f.; Bosworth, Toller 1898: 654; Lehmann 1986: 249f.). Zur Erweiterung der Verwandtschaftsterminologie durch Bezeichnungen der Verwandten außerhalb der väterlichen Familie siehe (Lehmann 1968: 15-17).

Im lübischen Recht kommt das Wort nur im Plural maghe vor, manchmal mit dem Attribut nêgest 'nächst', vgl. de negesten maghe 'die nächsten Verwandten' (Ki 27), erer negesten maghe 'ihrer nächsten Verwandten' (Ki 229). Wie sonst im Mittelniederdeutschen wird damit die Bedeutung 'Blutsverwandter' wiedergegeben, vgl. mach 'Verwandter, besonders Seitenverwandter' (Lasch, Borchling 1956-2007, 2: Sp. 881).

vrunt (vrünt, vrint, vrent) (m.) 'Verwandter' (Ki 10, 15, 29, 114, 122f., 171f., 193, 201f., 219, 255, Ki, Register, Artikeltitel 10 und 206) - vgl. got. frijonds (m. -nd) von frijon (schw. V. 2) 'lieben' zu gr. $\varphi \imath \lambda o_{\varsigma}$ 'Freund', frijondi (f. - - jō) zu gr. $\varphi \imath \lambda \eta ́$ 'Freundin', an. 
frǽndi 'Verwandter', ae. frēōnd 'Freund', afries. friond, friund 'Freund', 'Verwandter', as. friund, ahd. friunt 'Freund', vgl. aind. priyāyáte 'sich befreunden', aksl., aruss. приьлти 'lieb haben, mögen', прицмель 'Freund, Vertrauter, Geselle', прицзнь 'Freundlichkeit, Wohlwollen' und weitere indogermanische Parallelen (Cleasby, Vigfusson 1957: 176f.; Bosworth, Toller 1898: 335; von Richthofen 1840: 766f.; Lehmann 1986: 128f.). Zum Bedeutungswandel im Skandinavischen siehe (Cleasby, Vigfusson 1957: 176). Die Bedeutung 'Verwandter' ist auch im Altfriesischen belegt, besonders in Zusammensetzungen, vgl. federfriund 'Verwandter väterlicherseits', mōderfriund 'Verwandter mütterlicherseits' (von Richthofen 1840: 730, 766f.)

Im Mittelniederdeutschen kann das Wort vrünt sowohl 'persönlicher Freund, Vertrauter, Geselle, Anhänger, Getreuer' als auch 'Verwandter, Blutsverwandter (nicht in direkter Linie)' bedeuten (Lasch, Borchling 1956-2007, 2: Sp. 1016f.). Im Kontext des lübischen Rechts ist mnd. vrunt (vrünt, vrint, vrent) ausschließlich in der Bedeutung 'Verwandter' belegt, vgl. Welic wedewe oder iuncfruwe. svnder erer vrunde rat wil man nemen (Ki 10, Artikeltitel van echtschap 'Von der Ehe'), Steruet eneme manne de kindere heuet. sin wif. vnde nimt he ene andere. so schal he rekeninge holden. den vrunden sinder kindere. (Ki 114, Artikeltitel van dode 'Vom Tod'). Zu den Varianten vrunt (vrünt). vrint, vrent in den Lübecker Rechtscodizes siehe (Korlén 1951: 55f.; Peters 2012: 352; Ganina 2017: 183), wobei die Minderheitsvariante vrint als eine westliche Spur in der jüngeren Artikelreihe des Kieler Codex (Art. 170-239) interpretiert wird (Peters 2012: 352) und im Bardewikschen Codex als Minderheitsvariante vorkommt, und zwar vrunt mit Ableitungen (10x) gegenüber vrint/vrinde (3x) und vrende, vrende, uerint (je 1x) (Ganina 2017: 183).

\section{Fazit}

Die Untersuchung hat gezeigt, dass das lübische Recht über das entwickelte System der Verwandtschaftsbezeichnungen verfügt, das die altgermanische bzw. westgermanische Weiterentwicklung des indogermanischen Systems repräsentiert. Als nächste Entsprechung dafür kommt die ingwäonische Verwandtschaftsterminologie vor, die im Altenglischen und ggf. im Altfriesischen belegt ist. Im Vergleich zum sonstigen Korpus des Mittelniederdeutschen werden die Verwandtschaftsbezeichnungen im lübischen Recht in ihren ursprünglichen archaischen Bedeutungen gebraucht, wobei der Belegbestand durch entsprechende Rechtskollisionen bedingt wird. 


\section{Literatur}

Benecke, G. F., Müller W., Zarncke F., 1854-1866: Mittelhochdeutsches Wörterbuch. 3 Bde. Leipzig.

Ebel, W., 1980: Erbe, Erbgut und wohlgewonnen Gut im lübischen Recht. Zeitschrift der Savigny-Stiftung für Rechtsgeschichte. Germanistische Abteilung. 97, 1-42.

Bosworth, J., Toller, T. N., 1898: An Anglo-Saxon dictionary: Based on the manuscript collections of the late J. Bosworth. Oxford.

Cleasby R., Vigfusson, G., 1957: An Icelandic-English dictionary. 2. Aufl. Oxford.

Ebel, W., 1971: Lübisches Recht. Bd. I. Lübeck.

Ganina, N., 2017: Zur Schreibsprache des Lübischen Rechts. Indoeuropäische Sprachwissenschaft und klassische Philologie. [Индоевропейское языкознание и классическая филология]. 21, 174-184.

Haubrichs, W., 2001: Die Erfindung der Enkel: Germanische und deutsche Terminologie der Verwandtschaft und der Generationen. Lili Zeitschrift fur Literaturwissenschaft und Linguistik. 120 (4), 41-80.

Kluge, F., 1999: Etymologisches Wörterbuch der deutschen Sprache. 23., erw. Aufl., bearb. von E. Seebold. Berlin; New York.

Korlén, G., 1951: Norddeutsche Stadtrechte. Bd. II: Das mittelniederdeutsche Stadtrecht von Lübeck nach seinen ältesten Formen. Lund; Kopenhagen (Lunder Germanistische Forschungen 23).

Lasch, A., Borchling, C., 1956-2007: Mittelniederdeutsches Handwörterbuch. Hrsg. nach G. Cordes und A. Hübner ab 1993 von D. Möhn und I. Schröder. 3 Bde. Neumünster.

Lehmann, W. P., 1968: The Proto-Germanic words inherited from ProtoIndo-European which reflect the social and economic status of the speakers. Zeitschrift für Mundartforschung. 35, 1-25.

Lehmann, W. P., 1986: A Gothic etymological dictionary: Based on the third edition of 'Vergleichendes Wörterbuch der gotischen Sprache' by Sigmund Feist. Leiden.

Lexer, M., 1872-1878: Mittelhochdeutsches Handwörterbuch. 3 Bde. Leipzig.

Nikolaus von Jeroschin, 1861: Di Kronike von Pruzinlant des Nicolaus von Jeroschin. Hg. von E. Strehlke. In: Scriptores Rerum Prussicarum. Die Geschichtsquellen der preußischen Vorzeit bis zum Untergange der Ordensherrschaft. Hg. von T. Hirsch, M. Töppen, E. Strehlke. Leipzig (Nachdruck Frankfurt a.M. 1965). Bd. 1. S. 291-648.

Peters, R., 2012: Die Kanzleisprache Lübecks. Kanzleisprachenforschung. Ein internationales Handbuch. Hg. von A. Greule, J. Meier und A. Ziegler [u.a.]. Berlin, 347-365.

Richthofen, K. von, 1840: Altfriesisches Wörterbuch. Göttingen (Neudruck Aalen 1970).

Schiller, K., Lübben, A. 1875-1881: Mittelniederdeutsches Wörterbuch. 6 Bde. Bremen.

Toller, T. N., 1921: An Anglo-Saxon dictionary: Based on the manuscript collections of the late J. Bosworth. Supplement. Oxford.

Vries, J. de, 1962: Altnordisches etymologisches Wörterbuch. 2. verb. Aufl. Leiden. 